\title{
Cardiovascular System Findings Character Result in Standard Format
}

National Cancer Institute

\section{Source}

National Cancer Institute. Cardiovascular System Findings Character Result in Standard

Format. NCl Thesaurus. Code C124015.

The standard character or string for representation and reporting of cardiovascular system findings data. 